Research Article

\title{
Exact Solutions for the Conformable Space-Time Fractional Zeldovich Equation with Time-Dependent Coefficients
}

\author{
Sami Injrou \\ Department of Mathematics, Faculty of Sciences, Tishreen University, Lattakia, Syria \\ Correspondence should be addressed to Sami Injrou; s.injrou@tishreen.edu.sy
}

Received 26 February 2020; Accepted 15 April 2020; Published 12 May 2020

Academic Editor: Giovanni P. Galdi

Copyright (c) 2020 Sami Injrou. This is an open access article distributed under the Creative Commons Attribution License, which permits unrestricted use, distribution, and reproduction in any medium, provided the original work is properly cited.

The aim of this paper is to improve a sub-equation method to solve the space-time fractional Zeldovich equation with timedependent coefficients involving the conformable fractional derivative. As result, we obtain three families of solutions including the hyperbolic, trigonometric, and rational solutions. These solutions may be helpful to explain several phenomena in chemistry, including the combustion process. The study shows that the used method is effective and reliable and can be utilized as a substitution to construct new solutions of different types of nonlinear conformable fractional partial differential equations (NFPDEs) with variable coefficients.

\section{Introduction}

Though the concept of fractional derivative is more than 300 years old, in the last and present two centuries, the role of fractional calculus has been increasing because of its application area in various domains including the diffusion of biological population, signal processing, plasma physics, optical fiber, chemical kinematics, solid state physics, electrical network, finance, fluid flow, and control theory [1-6]. In particular, the noninteger order models have proven to be very useful to describe numerous scales, namely, nanoscale, microscale, and mesoscale. As a result of interest, the fractional differential equations (FDEs) have attracted the attention of many researchers. Several mathematicians proposed various types of fractional derivatives. The most popular ones are Riemann-Liouville, Caputo, Grunwald-Letnikov, Hadamard, Erdelyi, Kober, Marchaud, and Riesz [7-10]. Most types of fractional derivatives do not satisfy the classical formulas of derivative such as product rule, quotient rule, and chain rule (see [11] for example).

Recently, a new fractional derivative based on a limit form as in usual derivative has been introduced by Khalil et al. [12] called conformable fractional derivative. One can see that it is theoretically much easier to handle and satisfies the classical properties mentioned above. Because of the importance of the exact solutions of the nonlinear fractional partial differential equations (NFPDEs) to understand the nonlinear physical or chemical phenomena, many researchers solved NFPDEs with conformable derivative, such as that given in Aminikhah et al. [13] who used the sub-equation method to find the exact solutions to the fractional $(1+1)$ and $(2+1)$ regularized long-wave equations. Cenesiz and Kurt [14] obtained exact solutions to the space fractional advection diffusion equation and the space fractional telegraph equation by introducing conformable fractional complex transform. The Kudryashov method and the modified extended tanh expansion method with the Riccati differential equation were employed to establish various types of analytical solutions to the conformable space-time fractional Benney-Luke equation by Khalid and Nuruddeen [15], Al-Shawbal et al. [16] applied the two variables $\left(G^{\prime} / G, 1 / G\right)$-expansion method to obtain solitary and periodic wave solutions of higher-dimensional conformable time-fractional differential equations, such as $(2+1)$-dimensional time-fractional biological population model and nonlinear $(3+1)$ dimensional KdV-Zakharov-Kuznetsov equation. Exact solutions of some conformable fractional equations in the RLW-class, such as the conformable time-fractional RLW 
equation, the conformable time-fractional mRLW equation, and the conformable time-fractional sRLW equation, are investigated by using Sine-Gordon expansion approach by Korkmaz et al. [17]. Koyunlu et al. [18] found topological 1-soliton solutions to time-fractional modified EW equation and time-fractional Klein-Gordon equation by hyperbolic function ansatz method. Eslami and Talaghani [19] used the differential transform method to solve several conformable time-fractional partial differential equations. The exp-function method and perturbationiteration algorithm were used by Kurt et al. [20] to obtain some analytical and approximate solutions to fractional coupled Burgers' equation. More recently, Tozar et al. [21] utilized a new extended direct algebraic method to obtain a wide set of solutions to the fractional Camassa-Holm equation. Kurt [22] obtained new analytical and numerical results for $(2+1)$-dimensional time-fractional Bogoyavlensky-Konopelchenko equation by using the sub-equation method and the residual power series method.

Latterly, the study of NFPDEs with time-dependent coefficients has caught much more attention due to their ability to describe many physical or chemical phenomena in a realistic way than the constant coefficient ones. Zhang et al. [23] introduced a method based on the homogeneous balance principle using fractional Ricatti differential equation called fractional sub-equation method to find traveling wave solutions to NFPDEs.

In this paper, we propose an improved sub-equation method and use a traveling wave transformation in order to reduce a NFPDE to an ODE.

The advantages of the proposed method are its reliability and the reduction in the size of computational domain, and it needs less computation with respect to other analytical methods such as exp-function method or $\left(G^{\prime} / G, 1 / G\right)$-expansion method that require many complicated computations, especially, if the coefficients in the predicted solution are variable. Moreover, our method gives a wide set of different types of solutions.

As an application, we will use this method to look for the exact solutions of the space-time fractional Zeldovich equation with time-dependent coefficients in sense of conformable fractional derivative:

$$
{ }_{t} T_{\alpha} u+p(t){ }_{x x} T_{2 \alpha} u+q(t) u^{2}+r(t) u^{3}=0,
$$

where $\alpha \in(0,1), u=u(x, t)$ is an unknown function, and $p(t), q(t)$, and $r(t)$ are real-valued functions. The Zeldovich equation with integer order describes the combustion process, where $u(x, t)$ represents the temperature [24]. ${ }_{t} T_{\alpha}$ and ${ }_{x} T_{\alpha}$ are the space and time conformable fractional derivatives, respectively. For $\alpha=1, p(t)=-1, q(t)=-1$, and $r(t)=1$, equation (1) will be reduced to the standard Zeldovich equation.

\section{Preliminary}

In this section, we present the definition of conformable fractional derivative and its important properties. Also, we describe the main steps of the proposed method.

\subsection{Conformable Fractional Derivative}

Definition 1 (see [12]). Given a function $f:[0, \infty) \longrightarrow \mathbb{R}$, then the conformable fractional derivative of $f$ of order $\alpha$ is defined by

$$
T_{\alpha}(f)(t)=\lim _{\varepsilon \longrightarrow \infty} \frac{f\left(t+\varepsilon t^{1-\alpha}\right)-f(t)}{\varepsilon},
$$

for all $t>0$ and $\alpha \in(0,1)$. If $f$ is $\alpha$-differentiable in some $(0, a)$ for $a>0$ and $\lim _{t \rightarrow 0^{+}} f^{(\alpha)}(t)$ exists, then define $f^{(\alpha)}(0)=\lim _{t \longrightarrow 0^{+}} f^{(\alpha)}(t)$.

Theorem 1 (see [12]). Let $\alpha \in(0,1]$ and $f$ and $g$ be $\alpha$-differentiable at a point $t>0$. Then,

(1) $T_{\alpha}(a f+b g)=a T_{\alpha}(f)+b T_{\alpha}(g)$, for all $a, b \in \mathbb{R}$

(2) $T_{\alpha}\left(t^{p}\right)=p t^{p-\alpha}$, for all $p \in \mathbb{R}$

(3) $T_{\alpha}(\lambda)=0$, for all constant functions $f(t)=\lambda$

(4) $T_{\alpha}(f \cdot g)=f T_{\alpha}(g)(t)+g T_{\alpha}(f)$

(5) $T_{\alpha}(f / g)=\left(g T_{\alpha}(f)-f T_{\alpha}(g) / g^{2}\right)$

(6) If, in addition, $f$ is differentiable, then $T_{\alpha}(f)(t)=$ $t^{1-\alpha}(d f / d t)(t)$

Theorem 2 (see [25]). Let $f:[0, \infty) \longrightarrow \mathbb{R}$ be a function such that $f$ is differentiable and also $\alpha$-differentiable. Let $g$ be a function defined in the range of $f$ and also differentiable; then,

$$
{ }_{t} T_{\alpha}(f \cdot g)(t)=t^{1-\alpha} g^{\prime}(t) \cdot f^{\prime}(g(t)) \text {. }
$$

2.2. Description of the Method. Consider a NFPDE in two independent variables $x$ and $t$ as follows:

$$
P\left(u, T_{\alpha} T_{\alpha} u{ }_{x} T_{\alpha} u, \ldots\right)=0 ; \quad 0<\alpha \leq 1,
$$

where $u(x, t)$ is an unknown function and $P$ is a polynomial in $u$ and its conformable fractional partial derivatives.

We summarize the sub-equation method [23] as follows:

Step 1. Using the generalized traveling wave transformation,

$$
\begin{aligned}
u(x, t) & =u(\zeta) \\
\zeta & =k \frac{x^{\alpha}}{\alpha}+\int t^{\alpha-1} c(t) \mathrm{d} t+\delta,
\end{aligned}
$$

where $k$ and $\delta$ are constants and $c(t)$ is a continuous function. This transformation can reduce NFPDE (4) to an ODE with respect to one independent variable $\zeta$ so,

$$
Q\left(u, u^{\prime}, u^{\prime \prime}, \ldots\right)=0,
$$

where $Q$ is a polynomial in $u$ and its derivatives.

Step 2. We suppose the predicted solutions to (6) of the following form: 


$$
u(\zeta)=\sum_{j=-n}^{n} a_{j}(t) \phi^{j}(\zeta)
$$

where $a_{j}(t)(j=-n,-n+1, \ldots, n-1, n)$ are all functions of $t$ to be determined later. We determine the positive integer $n$ by considering the homogeneous balance between the highest-order derivatives and nonlinear terms appearing in (6). $\phi=\phi(\zeta)$ satisfies the following Riccati equation:

$$
\phi^{\prime}=\sigma+\phi^{2}
$$

where $\sigma$ is a constant. One can found that the solutions of Riccati equation (8) are given by [26]

$$
\phi(\zeta)= \begin{cases}-\sqrt{-\sigma} \tanh (\sqrt{-\sigma} \zeta) ; & \sigma<0, \\ -\sqrt{-\sigma} \operatorname{coth}(\sqrt{-\sigma} \zeta) ; & \sigma<0, \\ \sqrt{\sigma} \tan (\sqrt{\sigma} \zeta) ; & \sigma>0, \\ -\sqrt{\sigma} \cot (\sqrt{\sigma} \zeta) ; & \sigma>0, \\ -\frac{1}{\zeta+\mu} ; & \sigma=0,\end{cases}
$$

where $\mu$ is a constant.

Step 3. Substituting (7) and (8) into (6), collecting the coefficients of $[\phi(\zeta)]^{j}$, and then setting the coefficients of $[\phi(\zeta)]^{j}$ to be zero, we get an overdetermined system of nonlinear differential equations for $a_{j}(t)$ and $\zeta$.

Step 4. Solving the overdetermined system in Step 3 for $a_{j}(t)$ and $\zeta$ and then using these results and the solutions of (8) in (7), one can obtain the explicit solutions of (6). Finally, using transformation (5), one get the exact solutions of (4).

\section{Main Results}

Let us consider equation (1). Using generalized traveling wave transformation (5) and Theorem 1 and Theorem 2, equation (1) can be reduced to the following nonlinear ordinary differential equation:

$$
c(t) u^{\prime}+k^{2} p(t) u^{\prime \prime}+q(t) u^{2}+r(t) u^{3}=0,
$$

where $u^{\prime}=(d u / d \zeta)$ and $u^{\prime \prime}=\left(d^{2} u / d \zeta^{2}\right)$. We suppose that equation (10) admits a solution by the form of (7). Balancing the highest order derivative term $u^{\prime \prime}$ with nonlinear term $u^{3}$, one can find $n=1$. So,

$$
u(\zeta)=a_{0}(t)+a_{1}(t) \phi(\zeta)+\frac{a_{-1}(t)}{\phi(\zeta)} .
$$

Substituting (11) and (8) into (10), collecting all the terms with the same power of $[\phi(\zeta)]^{j}(j=-3,-2, \ldots, 2,3)$ and then equating each the coefficient of $[\phi(\zeta)]^{j}(j=-3,-2, \ldots, 2,3)$ with zero, one can obtain the overdetermined system of nonlinear differential equations for $a_{0}(t), a_{1}(t), a_{-1}(t)$, and $c(t)$ :

$$
\begin{array}{rl}
\phi^{0}: & 6 r(t) a_{0}(t) a_{1}(t) a_{-1}(t)+a_{-1}(t) c(t)+q(t) a_{0}^{2}(t)+r(t) a_{0}^{3}(t) \\
& +2 q(t) a_{1}(t) a_{-1}(t)+\sigma a_{1}(t) c(t)+t^{1-\alpha} \frac{d a_{0}(t)}{d t}=0, \\
\phi: 2 & 2 q(t) a_{0}(t) a_{1}(t)+3 r(t) a_{0}^{2}(t) a_{1}(t)+3 r(t) a_{1}^{2}(t) a_{-1}(t) \\
& +2 \sigma k^{2} p(t) a_{1}(t)+t^{1-\alpha} \frac{d a_{1}(t)}{d t}=0, \\
\phi^{2}: & 3 r(t) a_{0}(t) a_{1}^{2}(t)+a_{1}(t) c(t)+q(t) a_{1}^{2}(t)=0, \\
\phi^{3}: 2 & 2 k^{2} p(t) a_{1}(t)+r(t) a_{1}^{3}(t)=0, \\
\phi^{-1}: & 2 k^{2} \sigma a_{-1}(t)+2 q(t) a_{0}(t) a_{-1}(t)+3 r(t) a_{0}^{2}(t) a_{-1}(t) \\
& +3 r(t) a_{1}(t) a_{-1}^{2}(t)+t^{1-\alpha} \frac{d a_{-1}(t)}{d t}=0, \\
\phi^{-2}: & \sigma a_{-1}(t) c(t)+3 r(t) a_{0}(t) a_{-1}^{2}(t)+q(t) a_{-1}^{2}(t)=0, \\
\phi^{-3}: & 2 \sigma^{2} k^{2} a_{-1}(t)+r(t) a_{-1}^{3}(t)=0 .
\end{array}
$$

Solving this system, we obtain four cases. For simplicity, we introduce the notation $\eta(t)=\exp \left(-\int t^{\alpha-1} f(t) \mathrm{d} t\right)$, where $f(t)$ is a continuous function to obtain the following cases. 
Case 1

$$
\begin{aligned}
a_{0}(t) & =\eta(t), \\
a_{-1}(t) & =0, \\
a_{1}(t) & =c_{1} \exp \left[-\int\left(2 q(t) \eta(t)+3 r(t) \eta^{2}(t)+2 \sigma k^{2} p(t)\right) t^{\alpha-1} \mathrm{~d} t\right], \\
c(t) & =\frac{-1}{\sigma a_{1}(t)} \eta(t)\left(q(t) \eta(t)+r(t) \eta^{2}(t)-f(t)\right) .
\end{aligned}
$$

\section{Case 2}

Case 3

$$
\begin{aligned}
a_{0}(t) & =c_{3} \eta(t), \\
a_{1}(t) & =c_{4} \eta(t), \\
a_{-1}(t) & =\frac{\exp \left[-\int\left(2 q(t) c_{3} \eta(t)+3 r(t) c_{3}^{2} \eta^{2}(t)+2 \sigma k^{2}\right) t^{\alpha-1} \mathrm{~d} t\right]}{c_{5}+3 \int \exp \left[-\int\left(2 q(t) c_{3} \eta(t)+3 r(t) c_{3}^{2} \eta^{2}(t)+2 \sigma k^{2}\right) t^{\alpha-1} \mathrm{~d} t\right] r(t) c_{4} \eta(t) t^{\alpha-1} \mathrm{~d} t} \\
c(t) & =-\frac{c_{4} a_{-1}(t) \eta(t)\left(6 c_{3} r(t)+2 q(t)\right)+c_{3}^{2} q(t) \eta^{2}(t)+c_{3}^{3} r(t) \eta^{3}(t)-c_{3} f(t) \eta(t)}{a_{-1}(t)+c_{4} \sigma \eta(t)}
\end{aligned}
$$

Case 4

$$
\begin{aligned}
a_{0}(t) & =c_{3} \eta(t), \\
a_{1}(t) & =c_{4} \eta(t), \\
a_{-1}(t) & =\frac{q(t) \eta(t)\left(\sigma c_{4}^{2}-c_{3}^{2}\right)+c_{3} r(t) \eta^{2}(t)\left(3 \sigma c_{4}^{2}-c_{3}^{2}\right)+c_{3} f(t)}{c_{4}\left(3 c_{3} r(t) \eta(t)+q(t)\right)}, \\
c(t) & =c_{4} \eta(t)\left(3 c_{3} r(t) \eta(t)+q(t)\right),
\end{aligned}
$$

where $c_{1}, c_{2}, c_{3}, c_{4}$, and $k$ are the arbitrary constants.

Having obtained the expressions for $a_{0}(t), a_{1}(t), a_{-1}(t)$, and $c(t)$, we construct, when $\sigma<0$, a family of hyperbolic function solutions of equation (1) from Cases 1-4 as each $u(x, t)$ has a number:

$$
\begin{aligned}
u(x, t)= & a_{0}(t)-\sqrt{-\sigma} a_{1}(t) \tan h\left(\sqrt{-\sigma}\left(k \frac{x^{\alpha}}{\alpha}+\int t^{\alpha-1} c(t) \mathrm{d} t+\delta\right)\right) \\
& -\frac{a_{-1}(t)}{\sqrt{-\sigma}} \operatorname{coth}\left(\sqrt{-\sigma}\left(k \frac{x^{\alpha}}{\alpha}+\int t^{\alpha-1} c(t) \mathrm{d} t+\delta\right)\right), \\
u(x, t)= & a_{0}(t)-\sqrt{-\sigma} a_{1}(t) \operatorname{coth}\left(\sqrt{-\sigma}\left(k \frac{x^{\alpha}}{\alpha}+\int t^{\alpha-1} c(t) \mathrm{d} t+\delta\right)\right) \\
& -\frac{a_{-1}(t)}{\sqrt{-\sigma}} \tanh \left(\sqrt{-\sigma}\left(k \frac{x^{\alpha}}{\alpha}+\int t^{\alpha-1} c(t) \mathrm{d} t+\delta\right)\right) .
\end{aligned}
$$


Also when $\sigma>0$, we have a family of trigonometric function solutions of equation (1) from Cases $1-4$ as each $u(x, t)$ has a number:

$$
\begin{aligned}
& u(x, t)=a_{0}(t)+\sqrt{\sigma} a_{1}(t) \tan \left(\sqrt{\sigma}\left(k \frac{x^{\alpha}}{\alpha}+\int t^{\alpha-1} c(t) \mathrm{d} t+\delta\right)\right) \\
& +\frac{a_{-1}(t)}{\sqrt{\sigma}} \cot \left(\sqrt{\sigma}\left(k \frac{x^{\alpha}}{\alpha}+\int t^{\alpha-1} c(t) \mathrm{d} t+\delta\right)\right) \\
& u(x, t)=a_{0}(t)-\sqrt{\sigma} a_{1}(t) \cot \left(\sqrt{\sigma}\left(k \frac{x^{\alpha}}{\alpha}+\int t^{\alpha-1} c(t) \mathrm{d} t+\delta\right)\right) \\
& -\frac{a_{-1}(t)}{\sqrt{\sigma}} \tan \left(\sqrt{\sigma}\left(k \frac{x^{\alpha}}{\alpha}+\int t^{\alpha-1} c(t) \mathrm{d} t+\delta\right)\right) \text {. }
\end{aligned}
$$

One can also obtain, when $\sigma=0$, a family of rational solutions of equation (1) from Cases 1-4 as follows:

$$
\begin{aligned}
u(x, t)= & a_{0}(t)-\frac{a_{1}(t)}{k\left(x^{\alpha} / \alpha\right)+\int t^{\alpha-1} c(t) \mathrm{d} t+\lambda} \\
& -a_{-1}(t)\left(k \frac{x^{\alpha}}{\alpha}+\int t^{\alpha-1} c(t) \mathrm{d} t+\lambda\right),
\end{aligned}
$$

where $\lambda=\delta+\mu$.

\section{Conclusion}

In this work, we construct three types of exact solutions including hyperbolic function solutions, trigonometric function solutions, and rational solutions to the space-time fractional Zeldovich equation in sense of conformable fractional derivative with time-dependent coefficients by using the sub-equation method. The solutions obtained in this paper are recommended for future research in chemistry, namely, on the combustion models. Remarkably, these solutions and the proposed traveling wave transformation have not been reported in other literature. We might conclude that the used method in this paper is convenient and efficient and can be used for many NFPDEs involving conformable fractional derivative with time-dependent coefficients arising in physics and chemistry. It is worth to apply other algebraic methods to NFPDEs, such as expfunction method. This is our aim in the future.

\section{Data Availability}

No data were used to support this study.

\section{Conflicts of Interest}

The authors declare that they have no conflicts of interest.

\section{Acknowledgments}

This work was supported by Tishreen University.

\section{References}

[1] K. S. Miller and B. Ross, An Introduction to the Fractional Calculus and Fractional Differential Equations, Wiley, Hoboken, NJ, USA, 1993.

[2] I. Podlubny, Fractional Differential Equations, Academic, San Diego, CA, USA, 1999.

[3] F. S. Mozaffari, H. Hassanabadi, H. Sobhani, and W. S. Chung, "On the conformable fractional quantum mechanics," Journal of the Korean Physical Society, vol. 72, no. 9, pp. 980-986, 2017.

[4] W. S. Chung, S. Zare, and H. Hassanabadi, "Investigation of conformable fractional schrödinger equation in presence of killingbeck and hyperbolic potentials," Communications in Theoretical Physics, vol. 67, no. 3, pp. 250-254, 2017.

[5] W. S. Chung, S. Zarrinkamar, S. Zare, and H. Hassanabadi, "Scattering study of a modified cusp potential in conformable fractional formalism," Journal of the Korean Physical Society, vol. 70, no. 4, pp. 348-352, 2017.

[6] F. S. Mozaffari, H. Hassanabadi, H. Sobhani, and W. S. Chung, "Investigation of the dirac equation by using the conformable fractional derivative," Journal of the Korean Physical Society, vol. 72, no. 9, pp. 987-990, 2018.

[7] U. N. Katugampola, "New approach to a generalized fractional integral," Applied Mathematics and Computation, vol. 218, no. 3, pp. 860-865, 2011.

[8] U. N. Katugampola, "New approach to generalized fractional derivatives," Bulletin of Mathematical Analysis and Applications, vol. 6, no. 4, pp. 1-15, 2014.

[9] A. A. Kilbas, H. M. Srivastava, and J. J. Trujillo, Theory and Applications of Fractional Differential Equations, Elsevier, Amsterdam, Netherlands, 2016.

[10] S. G. Samko, A. A. Kilbas, and O. I. Marichev, "Fractional Integrals and Derivatives," Theory and Applications, Gordon and Breach, Yverdon, Switzerland, 1993.

[11] C.-s. Liu, "Counterexamples on Jumarie's two basic fractional calculus formulae," Communications in Nonlinear Science and Numerical Simulation, vol. 22, no. 1-3, pp. 92-94, 2015.

[12] R. Khalil, M. Al Horani, A. Yousef, and M. Sababheh, "A new definition of fractional derivative," Journal of Computational and Applied Mathematics, vol. 264, pp. 65-70, 2014.

[13] H. Aminikhah, A. H. Refahi Sheikhani, and H. Rezazadeh, "Sub-equation method for the fractional regularized longwave equations with conformable fractional derivatives," Scientia Iranica, vol. 23, no. 3, pp. 1048-1054, 2016. 
[14] Y. Cenesiz and A. Kurt, "New fractional complex transform for conformable fractional partial differential equations," Journal of Applied Mathematics, Statistics and Informatics, vol. 12, no. 2, 2016.

[15] K. A. Khalid and R. I. Nuruddeen, "Analytical treatment for the conformable space-time fractional benney-luke equation via two reliable methods," International Journal of Physical Research, vol. 5, no. 2, pp. 109-114, 2017.

[16] A. A. Al-Shawba1, F. A. Abdullah1, K. A. Gepreel, and A. Azmi, "Solitary and periodic wave solutions of higherdimensional conformable time-fractional differential equations using the $\left(G^{\prime} / G, 1 / G\right)$-expansion method," Advances in Difference Equations, vol. 362, 2018.

[17] A. Korkmaz, O. E. Hepson, K. Hosseini, H. Rezazadeh, and M. Eslami, "Sine-gordon expansion method for exact solutions to conformable time fractional equations in RLW-class," Journal of King Saud University-Science, vol. 32, no. 1, 2018.

[18] G. Koyunlu, G. Ahmad, J. S. Liman, and H. U. Muazu, "Topological 1-soliton solutions to fractional modified equal width equation and fractional klein-gordon equation," International Journal of Electrical, Electronics and Data Communication, vol. 6, no. 10, pp. 38-41, 2018.

[19] M. Eslami and S. A. Taleghani, "Differential transform method for conformable fractional partial differential equations," Iranian Journal of Numerical Analysis and Optimization, vol. 9, no. 2, pp. 17-29, 2019.

[20] A. Kurt, M. Şenol, O. Tasbozan, and M. Chand, "Two reliable methods for the solution of fractional coupled Burgers' equation arising as a model of polydispersive sedimentation," Applied Mathematics and Nonlinear Sciences, vol. 4, no. 2, pp. 523-534, 2019.

[21] A. Tozar, A. Kurt, and O. Tasbozan, "New wave solutions of time fractional integrable dispersive wave equation arising in ocean engineering models," Kuwait Journal of Science, vol. 47, no. 2, pp. 22-33, 2020.

[22] A. Kurt, "New analytical and numerical results for fractional bogoyavlensky-konopelchenko equation arising in fluid dynamics," Applied Mathematics-A Journal of Chinese Universities, vol. 35, no. 1, pp. 101-112, 2020.

[23] S. Zhang and H. Zhang, "Fractional sub-equation method and its applications to nonlinear fractional PDEs," Physics Letters A, vol. 375, no. 7, pp. 1069-1073, 2011.

[24] B. H. Gilding and R. Kersner, "Travelling waves in nonlinear diffusion convection reaction," in Progress in Nonlinear Differential Equations and Their Applications, Vol. 60, Springer, Berlin, Germany, 2004.

[25] T. Abdeljawad, "On conformable fractional calculus," Journal of Computational and Applied Mathematics, vol. 279, pp. 57-66, 2015.

[26] E. Fan, "Extended tanh-function method and its applications to nonlinear equations," Physics Letters A, vol. 277, no. 4-5, pp. 212-218, 2000. 